\title{
Crescimento econômico e instituições sob uma perspectiva evolucionária
}

\author{
Economic growth and institutions under an evolutionary perspective
}

\author{
Alexandre Schwinden Garcia', Adilson Giovanini", Pablo Felipe Bittencourt"'I
}

\begin{abstract}
Resumo
O objetivo deste estudo é promover a ampliação da compreensão sobre a relação entre o crescimento econômico e a atuação do Estado sob uma perspectiva evolucionária. Para isso, foi feita uma revisão de literatura buscando aspectos essenciais da teoria evolucionária capazes de explicar o crescimento econômico. Essa literatura mostra que as firmas, ao produzirem, são influenciadas por diferentes instituições e tecnologias sociais, de modo que a atividade produtiva demanda a coordenação de diferentes agentes. A mudança tecnológica não ocorre de forma contínua, estando sujeita a mudanças abruptas que demandam a adaptação das instituições e das tecnologias sociais. A capacidade de adaptação dos países às mudanças tecnológicas explica as maiores taxas de crescimento econômico apresentado por alguns países em detrimento dos demais. A presença de problemas de coordenação implica o surgimento de espaço para a atuação do Estado, o qual fica responsável por promover as mudanças institucionais e por dotar o sistema econômico de maior capacidade de adaptação, conseguindo assim promover o maior crescimento econômico.
\end{abstract}

Palavras-chave: Crescimento Econômico; Instituições; Mudança Institucional

\begin{abstract}
The objective of this study is to promote the broadening of the understanding of the relationship between economic growth and state performance under an evolutionary perspective. For this, a literature review was searched for essential aspects of evolutionary theory capable of explaining economic growth. This literature shows that firms, when producing, are influenced by different institutions and social technologies, so that productive activity demands the coordination of different agents. Technological change does not occur continuously and is subject to abrupt changes that require the adaptation of social institutions and technologies. The ability of countries to adapt to technological change explains the higher rates of economic growth presented by some countries to the detriment of others. The presence of coordination problems implies the emergence of space for State action, which is responsible for promoting institutional changes and for endowing the economic system with greater capacity for adaptation, thus achieving the promotion of greater economic growth.
\end{abstract}

Keywords: economic growth; institutions; institutional change.

\footnotetext{
Universidade Federal de Santa Catarina, SC, Brasil - alesg61@hotmail.com

II Universidade Federal de Santa Catarina, SC, Brasil - adilsoneconomia@gmail.com

IIIUniversidade Federal de Santa Catarina, SC, Brasil - pablofelipe.bittencourt@gmail.com
} 


\section{Introdução}

Dentre a vasta literatura que busca explicar o processo de crescimento econômico destaca-se o artigo seminal "An evolutionary theory of economic change", de Nelson e Winter (2005). Nesse artigo, os autores resgatam e aprofundam ideias e conceitos propostos pela teoria evolucionária de mudança econômica, que possui como foco de análise a firma. Esses autores retomaram as ideias propostas originalmente por Schumpeter (1934), argumentando que o motor do capitalismo e, assim, do crescimento econômico são as inovações tecnológicas, de modo que a compreensão do desempenho agregado da economia demanda a análise das variações predominantes por baixo do agregado, no nível das firmas (NELSON, 1995).

A observação das firmas de maneira desagregada evidencia uma variedade de comportamentos e respostas padrão a determinados problemas enfrentados por essas firmas diante do ambiente econômico. Esses comportamentos e respostas são os elementos da análise evolucionária, explicitados no conceito de rotinas, sendo as rotinas moldadas a partir do processo histórico de aprendizagem das firmas, de seus conhecimentos preestabelecidos e de seus sistemas de valores (NELSON; DOSI, 1994).

Entender o crescimento econômico e as inovações passa, portanto, por compreender as diferentes trajetórias de rotinas, utilizadas pelas firmas na produção de determinados bens. Essas rotinas transcendem à simples transformação de matérias-primas em produtos finais, assumindo natureza complexa, dinâmica e coevolutiva. Nelson e Sampat (2001) defendem a necessidade de considerar "how the activity is done", surgindo o conceito de "tecnologias sociais". Assim, a produção de uma firma é a composição de tecnologias físicas e sociais, isto é, um processo implícito.

As tecnologias sociais podem ser capacitadas ou restringidas por diferentes fatores, dentre eles, leis, normas, expectativas, estruturas de governança, mecanismos, costumes e modos de organização e de transação. Esses fatores podem sustentar e padronizar determinadas (e específicas) tecnologias sociais e, em contrapartida, podem tornar difíceis, inviáveis e não factíveis outras tecnologias, que são excluídas socialmente. Nesse sentido, Nelson (2008, p. 2) conceitua instituições como "[...] the factors and forces that mold and hold place social technologies".

Assim, as instituições, no contexto proposto por Nelson e Sampat (2001), são incorporadas a uma teoria evolucionária de crescimento econômico através de sua associação com o conceito de tecnologias sociais. Para tanto, Conceição (2012) destaca que se faz necessária a incorporação de alguns conceitos como rotinas e tecnologias sociais como inerentemente vinculados às instituições.

Essa introdução teve como objetivo elencar brevemente os diversos conceitos desenvolvidos pela literatura evolucionária. Conforme visto, a presente literatura caracteriza-se pela coexistência de diferentes conceitos e definições: inovações, rotinas, tecnologias sociais, firmas, instituições. Essa diversidade de conceitos, apresentada pela literatura evolucionária, se mostra virtuosa, evidenciando a acalorada discussão e a riqueza de ideias que caracterizam essa literatura. Porém, a coexistência de elevado número de conceitos, ora complementares, ora sobrepostos, acaba dificultando a realização de um debate mais preciso em torno do verdadeiro objetivo perseguido pela respectiva literatura, qual seja: os fatores responsáveis pelo crescimento econômico.

Grosso modo, a literatura evolucionária defende que o crescimento econômico é, em grande monta, explicado pelo desenvolvimento tecnológico. Afirmações como "o desenvolvimento tecnológico demanda o desenvolvimento das instituições adequadas" são comuns a essa literatura. O problema é que o termo "instituições" abarca uma diversidade de conceitos, assumindo diferentes significados. Como resultado, a afirmação supracitada se transforma em uma afirmação genérica, uma tautologia que pouco informa.

Dada essa abundância de conceitos e definições, este artigo possui como objetivo realizar uma revisão da literatura evolucionária, evidenciando os elos existentes entre os diferentes conceitos apresentados. Busca, assim, suprir uma lacuna existente na respectiva literatura, dado que essa carece de estudos que busquem revisitar esses diferentes conceitos, apresentando-os de forma simples, precisa, clara, direta e amigável ao leitor.

Ademais, este artigo dá um passo além, relacionando esses conceitos com a necessidade de políticas industriais voltadas para a promoção do desenvolvimento econômico. Busca desenvolver um argumento mais cirúrgico, preciso e lapidado, estabelecendo uma relação dos diferentes conceitos apresentados pela literatura evolucionária de forma mais acurada, de modo a identificar a linha de ação que pode ser adotada pelo Estado para, efetivamente, promover o crescimento econômico.

Além desta introdução, este artigo possui mais quatro seções. A segunda seção apresentará o referencial teórico, conferindo ênfase inicial à teoria evolucionária de crescimento. A terceira seção elencará os conceitos de instituições, mudanças institucionais e tecnologias sociais, preparando as bases para a seção 4. Esta seção discutirá como a estrutura institucional e as políticas públicas podem influenciar e coordenar as interações entre os agentes econômicos, promovendo o crescimento econômico. Dessa forma, será possível, na seção 5 , relacionar crescimento econômico e instituições sob uma perspectiva evolucionária. 


\section{Teoria evolucionária da firma}

A seguir, serão apresentados os elementos essenciais da teoria evolucionária com base na firma. Após essa etapa, a revisão de literatura se volta ao crescimento econômico sob a perspectiva evolucionária, que tem nas inovações sua força motriz.

\section{Quais são os elementos essenciais?}

Algumas características são essenciais quando se define uma teoria como evolucionária. Dosi e Nelson (1994) destacam duas delas: i) tem o propósito de explicar o movimento de algo no tempo ou porque algo está lá em determinado momento, sendo, portanto, uma análise essencialmente dinâmica; e ii) envolve elementos aleatórios que geram ou renovam alguma mudança nas variáveis em questão e mecanismos que selecionam sistematicamente a variação existente.

Quando aplicados ao contexto social, os processos evolucionários são imperfeitos, sendo caracterizados por uma dinâmica de "learning and discovering". Também existem mecanismos de seleção, emergindo a noção de "fitness" ou mais bem adaptado.

Com relação ao primeiro componente (processos dinâmicos de "learning and discovering"), Dosi e Nelson (1994) destacam que, contrariamente às teorias econômicas, que presumem que os atores possuem respostas ótimas para determinados contextos e problemas (abordagem neoclássica), na teoria evolucionária não é possível presumir que esses atores possuem boas respostas dadas, mas que essas ainda devem ser aprendidas.

O aprendizado dos atores é, portanto, fundamental na teoria evolucionária, sendo esse processo de aprendizado caracterizado por ser dependente de determinada trajetória (path-dependence): "Where they end up my depend to a considerable degree on how they got there" (DOSI; NELSON, 1994, p. 158). Assim, "a história conta", visto que os fundamentos dos comportamentos dos agentes envolvem um processo imperfeito de adaptação e descobertas erradas, onde os agentes paulatinamente testam e descobrem novas tecnologias e configurações, em um processo cronológico e dinâmico de tentativa e erro do qual emergem diferentes aprendizados.

A segunda característica guarda relação direta com processos de seleção de indivíduos dentro de uma população. Envolve a busca de uma resposta para a seguinte pergunta: o que considerar mais adaptado ou não ao meio, mais ou menos fit? A teoria evolucionária proposta por Nelson e Winter (2005) tem como foco principal a análise das firmas, as quais são mais ou menos "fit". Elas são motivadas pelos lucros e pela busca em aumentar esses lucros. As firmas são dotadas e modeladas como detentoras de certas capacidades limitadas e regras de decisão (rule-guided). Essas capacidades e regras podem ser modificadas em resposta à adoção de esforços na resolução de problemas e eventos aleatórios.

Nesse sentido, Dosi (1988) destaca que o esforço das firmas na resolução de problemas é a essência do processo de inovação tecnológica, que envolve o uso de informações extraídas em experiências já passadas pela firma, do conhecimento formal aprendido em sua trajetória e de capacidades específicas e não codificadas pelos inventores, ou seja, envolve conhecimentos com componentes tácitos. Dessa forma, a organização, dotada de determinada habilidade ou conhecimento, não é capaz de determinar com exatidão cada detalhe envolvido no processo, o qual se encontra "embodied" em rotinas possuídas pelas firmas.

Em suma, a análise evolucionária é essencialmente dinâmica e envolve aspectos de aprendizado e descoberta por parte dos atores. Isto posto, abre a possibilidade de adoção de políticas públicas influenciando as firmas na busca de novas trajetórias de conhecimentos, modificando, assim, as respostas dadas e as conduzindo para novas trajetórias com maior potencial de aprendizagem e desenvolvimento de inovações.

\section{Crescimento econômico para os evolucionários: a inovação como força motriz}

Nelson e Winter (2005) destacam que o coração da teoria evolucionária é o processo dinâmico em que os padrões de comportamento da firma e os resultados do mercado são determinados conjuntamente no tempo, podendo esse processo ser resumido da seguinte maneira: em determinado período as características operacionais da firma estabelecem a quantidade de insumos utilizados e o nível produção, sendo a oferta de insumos e as condições de demanda determinadas exogenamente. Contudo, as decisões provenientes das firmas determinam os preços dos insumos e dos produtos, proporcionando determinada rentabilidade.

A rentabilidade auferida afeta as decisões de investimento das firmas, o que determinará a sua capacidade de expansão, alterando o seu tamanho, o montante utilizado de insumos e o nível de produto, abrindo-se espaço para a obtenção de novos graus de rentabilidade e preços. Esse processo afeta a quantidade utilizada de insumo e o nível de produto gerado, mesmo que as caraterísticas operacionais das firmas se mantenham constantes. Contudo, dado que essas características mudam de acordo com os processos de pesquisa adotados pelas firmas, os processos de pesquisa e seleção se tornam simultâneos e interativos. 
Os preços, portanto, dão suporte ao processo de seleção e influenciam na direção da busca. Assim, pesquisa e seleção de novas rotinas evoluem conjuntamente ao longo do tempo, com a premissa, conforme destacam Nelson e Winter (2005), de que as condições da indústria em cada período plantam as sementes para as condições do próximo período. Nesse sentido, o processo de procura de novas rotinas e tecnologias é cumulativo: "What the firm can hope to do technologically in the future is constrained by what it has been capable of doing in the past" (DOSI, 1988, p. 1130).

Dosi e Nelson (1994) destacam que os modelos evolucionários podem explicar variações em nível agregado. Portanto, a mudança nas características das firmas pode ajudar a explicar por que diferentes países apresentam diferentes trajetórias de crescimento. Como destacado por Nelson (1995, p. 92): “[...] o desempenho do crescimento agregado da economia está fortemente relacionado às variações predominantes por baixo do agregado". Assim, a economia deve ser vista do desagregado para o agregado, e não o contrário.

Nesse sentido, o modelo de crescimento baseado na inovação tecnológica proposto por Nelson e Winter (2005) pode ser simplificado da seguinte forma: inovação tecnológica estocasticamente determinada gera lucros para a firma inovadora, o que faz com que ela aumente a formação de capital, obtendo maiores níveis de crescimento do seu faturamento. Posteriormente, o aumento da produtividade gera uma redução do uso de trabalho por produto, o qual é mais do que compensado pelo aumento no tamanho da firma, elevando-se a demanda por trabalho e, assim, os salários. Como resultado, a utilização de capital se torna mais barata em relação à utilização de trabalhadores, o que torna atrativa e lucrativa a procura por inovações que poupam trabalho. Aumenta-se, assim, o nível de capital intensivo da economia.

Como os salários reais, a produtividade do trabalho e o nível de capital da economia estão crescendo, o retorno do capital cai. Caso haja a criação de uma nova tecnologia produtiva, a taxa de lucros subirá novamente e esses lucros induzirão a uma nova rodada de investimento que aumentará novamente o nível de salários reais da economia. É importante destacar que a dinâmica econômica é baseada não somente em inovações de produtos, mas também em inovação de processos e das formas de organização das firmas.

Dosi (1988) destaca que cada paradigma tecnológico dominante implica um balanceamento entre determinantes exógenos e endógenos às firmas. Cada paradigma envolve, portanto, modos específicos de pesquisa, bases de conhecimento e combinação entre conhecimentos públicos e privados. Assim, Nelson e Winter (2005) argumentam que a habilidade de dirigir um carro (ou uma rotina) vai além de saber acelerar, frear ou passar a marcha, mas também envolve a habilidade de observar o ambiente à volta, como os demais veículos e as placas de sinalização.

Considerar, portanto, componentes exógenos à firma como fundamentais ao processo de inovação e desenvolvimento nos remete a observar o meio e os sinais que influenciam no comportamento das firmas, isto é, o ambiente institucional em sentido amplo. Ao vincular rotinas ou habilidades e tecnologias sociais como inerentes às instituições, é possível incorporar instituições à teoria evolucionária de crescimento econômico.

\section{Instituições, mudanças institucionais e tecnologias sociais}

O termo “instituições" é amplamente citado na literatura econômica. Porém, não há convergência de significado, sendo ele utilizado de forma genérica, assumindo diferentes sentidos. A contribuição de Nelson e Sampat (2001) para essa discussão é fundamental e marcante. Os autores defendem que, apesar da grande diversidade conceitual, grande parte da literatura destaca que instituições são fatores que suportam as maneiras com que os diferentes agentes agem e interagem, determinando, assim, o que será atingido. Sugerem, então, o conceito de "tecnologias sociais" como o mais adequado para representar esses fatores.

De maneira geral, atividades econômicas envolvem diversos atores e fatores para atingir determinados objetivos. Nelson e Sampat (2001) observam que uma receita ou determinada tecnologia, com todos os ingredientes e fatores necessários, é "muda" em relação à forma como a receita ou tecnologia é feita, isto é, qualquer tecnologia requer algum grau de coordenação para atingir determinado objetivo, sejam os passos a seguir ou a ordem dos ingredientes. Os autores propõem dividir uma tecnologia em dois aspectos: os aspectos físicos (tecnologias físicas) e os aspectos relacionados ao modo como o trabalho ou a receita é dividida e coordenada (tecnologias sociais).

Nesse contexto, cabe destacar uma das características-chaves das rotinas que fazem da coordenação entre os diversos atores fundamental: o fato de serem amplamente conhecidas e empregadas por especialistas de determinada área e possuírem elementos padrão largamente utilizados, sendo culturalmente compartilhados.

Nelson e Sampat (2001) defendem que o domínio desses aspectos culturais compartilhados é fundamental para as organizações em dois aspectos. Primeiro, em diferentes processos produtivos - desde receitas de bolo, até o modo de fazer semicondutores -, a rotina é resultado de contribuições de diversas partes, sendo essas diferentes contribuições a razão central da sua efetividade. Segundo, as rotinas são parte de um sistema de rotinas que dependem, de alguma maneira, de atores externos à organização, sendo mais fácil, por exemplo, obter ajuda de atores que sabem o que é necessário fazer para atingir determinado objetivo. 
Virtualmente, todas as atividades econômicas envolvem tanto tecnologias físicas como sociais, ou seja, associadas com um vetor de insumos e produtos, mas também com um processo implícito. Nelson e Winter (2002) observam que no livro The Wealth of Nations Adam Smith destacava a importância tanto dos aspectos físicos da produção tecnológica quanto da organização da forma de trabalho para que o processo produtivo fosse levado a cabo com êxito, reforçando, assim, a ideia de coevolução entre tecnologias físicas e sociais.

As tecnologias sociais são, portanto, "[...] enable and constrained by things like laws, norms, expectations, governing structures and mechanisms, customary modes of organizing and transactions" (NELSON, 2008, p. 3). A partir daí, Nelson (2008, p. 2) conceitua instituições como "[...] the factors and forces that mold and hold place social technologies".

Essas instituições, portanto, tendem a suportar e padronizar certas tecnologias sociais e fazer de outras inviáveis em determinada sociedade. Porém, nem todas as tecnologias sociais são instituições, apenas as que se tornam um padrão ou algo esperado a se fazer em determinada situação. A padronização de algumas tecnologias sociais também pode restringir determinadas rotinas dentro das organizações. Assim, a capacidade de aprender e escolher novas rotinas de forma deliberada pode ser dificultosa: "Routines do not eliminate choice, but they sharply channel it" (NELSON; SAMPAT, 2001, p. 42).

Nelson (2008) propõe que, ao se estudar crescimento econômico guiado por inovações, se deve então pensar esse processo como a coevolução de tecnologias sociais e físicas, e não apenas como resultado da última. Assim, a dinâmica institucional toma grandes proporções. $\mathrm{O}$ autor destaca o fato de que instituições geralmente apresentam estabilidade e rigidez.

Contudo, os avanços tecnológicos e o crescimento econômico podem mudar de forma abrupta tecnologias físicas e sociais. Em outras palavras, ainda que a mudança em determinada tecnologia física e social ocorra, em geral, de forma contínua, por meio de mudanças incrementais ao longo de uma trajetória tecnológica relativamente bem compreendida pelos envolvidos, a mudança pode também ser disruptiva e de grandes proporções.

Nesse sentido, Freeman e Perez (1988) e Perez (2004) destacam que grandes mudanças tecnológicas ou "paradigmas tecnoeconômicos" que impulsionam a transformação na forma de produzir em um amplo conjunto de setores (senão todos) são ocasionadas por revoluções tecnológicas, que, ao gerarem as grandes ondas de desenvolvimento, carregam as sementes de grandes transformações na sociedade, inclusive crises de ajustamento, períodos em que mudanças sociais e institucionais são necessárias para trazer um melhor "match" entre o novo regime tecnológico que emerge e a economia.

Assim, grandes mudanças tecnológicas trazem a necessidade de novo design institucional. A mudança institucional necessária é, contudo, lenta, podendo então "[...] drags on economic productivity and progressiveness" (NELSON, 2008, p. 2). Portanto, a mudança institucional é parte integrante do processo de desenvolvimento econômico (NELSON, 2006), podendo esse processo de mudança institucional também envolver tentativas, falhas e novas tentativas, ocorrendo aprendizado de acordo com os erros passados. Trata-se de um processo evolucionário e cultural (NELSON; SAMPAT, 2001).

Conceição (2009) destaca que, como o crescimento e o avanço tecnológico ocorrem na presença de elevado nível de incerteza, as instituições podem ser contraprodutivas caso não haja coordenação e adoção de estratégias adequadas com relação à orientação do paradigma tecnológico. Isso traz à discussão a questão das diferenças no tipo de mudança institucional necessária ao desenvolvimento em diferentes contextos. Nelson (2008) pondera que diferentes tempos requerem diferentes instituições de suporte e, assim, novas tecnologias sociais. Contudo, países bem-sucedidos em determinado período são aqueles que já possuem ou que têm a capacidade de construir novas instituições de forma rápida e correta.

Resumidamente, esta seção procurou mostrar que a mudança institucional contribui para o desenvolvimento de tecnologias sociais e físicas, responsáveis por impulsionar o desenvolvimento econômico, sendo elemento fundamental à análise das diferentes trajetórias econômicas apresentadas pelos países. A mudança institucional é elemento básico à compreensão dos determinantes da mudança tecnológica. Assim, Nelson (2008) cria uma ponte entre avanços tecnológicos e instituições, visto que instituições são estruturas que suportam as tecnologias sociais e estas, junto com as físicas, são igualmente importantes no entendimento do processo de inovação, responsável por promover o crescimento econômico. Nelson (2002, p. 27) pontua: "[...] this coevolutionary process is the driving force behind economic growth".

\section{Estrutura institucional e política industrial: o Estado como coordenador das interações entre os agentes}

Na seção anterior se destacaram as tecnologias sociais como elementos analíticos fundamentais à compreensão dos limites e das possibilidades do avanço tecnológico. As tecnologias sociais se encontram enraizadas na sociedade e são sustentadas por suas instituições, sofrendo a influência da mudança tecnológica, a qual, por meio da concorrência ou do surgimento de inovações, pode tornar parte das tecnologias sociais obsoleta. Mais do que isso, 
procurou-se trazer para a discussão o papel da mudança em nível agregado, o qual está cristalizado nas noções de ciclos longos de Freeman e Perez (1988) e Perez (2004), principalmente ao ressaltarem aspectos da mudança institucional que se combinam à mudança tecnológica historicamente.

Resumidamente, a mudança está no centro da análise tanto no nível micro (das tecnologias físicas e sociais) como no nível macro (das revoluções tecnológicas e dos paradigmas tecnoeconômicos subjacentes). Adicionalmente, no final da seção anterior chamamos a atenção para a compreensão de Nelson (2008), para quem os países bem-sucedidos são os que guardam em suas sociedades as melhores condições para construir novas instituições e encaixar-se (to fit) a novas condições. O fato estilizado mais eloquente sobre essa interpretação é o conjunto limitado de países que se mantém com um nível mais elevado de renda per capita relativa com o passar dos anos.

De qualquer forma, isso traz à tona a pergunta: quando existe a necessidade de políticas públicas voltadas a coordenar as estratégias que possam aproveitar a mudança de paradigma? Cimolli et al. (2007) destacam que discussões em torno de políticas devem ser pautadas por questionamentos em torno da suficiência dos arranjos institucionais.

Chaminade et al. (2009) argumentam que a abordagem de falhas sistêmicas se coloca como fator preponderante para se avaliar a necessidade de políticas em determinados sistemas de inovação. Destacam que "While the market failure approach is about getting the prices right, the systemic approach is about getting the institutions right" (CHAMINADE et al., 2009, p. 5). Assim, o ponto de partida das discussões sobre políticas públicas deve ser sobre quais instituições podem levar a trajetórias inovativas.

Como determinadas tecnologias sociais podem ser vistas como instituições, estas podem ser responsáveis por controlar externalidades, conformar padrões de atividades inovativas, estruturas de incentivos subjacentes, investimentos, propensão a poupar, capacitação de mão de obra e competências (CIMOLLI et al., 2007). Assim, as instituições governam externalidades e complementaridades, administrando as regras de interação entre os agentes, moldando crenças e determinando as informações a que esses agentes têm acesso, e influenciando em suas normas de comportamento, sendo a coordenação um dos papéis fundamentais entre agentes heterogêneos.

Para melhor coordenar e observar as necessidades dos diversos agentes econômicos, é necessário organizar e verificar o entrelaçamento entre esses em torno de sistemas de inovação. Esse é um passo importante para um adequado desenho institucional.

Decorrente da heterogeneidade dos atores envolvidos, políticas públicas devem levar em consideração características específicas de cada sistema, a sua evolução e o contexto socioeconômico em que esse sistema se encontra inserido. Assim, uma adequada política de inovação deve levar em conta dois fatores: especificidades do sistema de inovação e um grande grau de experimentação. Destaca-se que "[...] in practical terms, what might be a problem in one system might not be a problem at all in another system" (CHAMINADE et al., 2009, p. 5).

Portanto, é importante destacar que diferentes graus de maturidade dos sistemas de inovação podem gerar diferentes demandas para os agentes envolvidos e, assim, diferentes intervenções em termos de política e estrutura institucional. A Figura 1 demonstra as diferenças entre sistemas de inovação emergentes e sistemas de inovação maduros.

Figura 1 - Sistemas de inovação emergentes e sistemas de inovação maduros

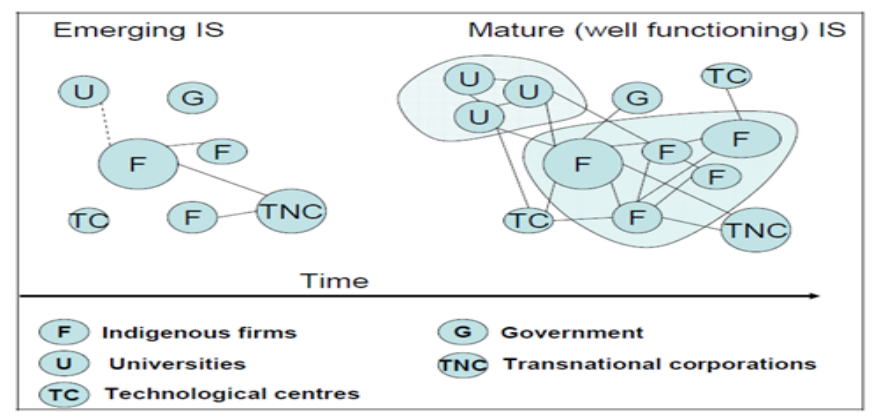

Fonte: Chaminade e Vang (2008).

As relações entre os agentes dos sistemas emergentes são fracas, quando comparadas com sistemas maduros. Porém, conforme observam Chaminade et al. (2009), a questão fundamental não é se as relações são fracas ou fortes, mas quais são os elementos críticos para o desenvolvimento de um sistema de inovação socialmente inclusivo e como as políticas de inovação podem ser desenhadas.

No que tange aos sistemas de inovação de países em desenvolvimento, Chaminade et al. (2009) destacam que, em sistemas emergentes, as capacitações cruciais para os agentes são aquelas relacionadas à capacidade de absorção e adaptação de tecnologias de uma fonte externa de conhecimento. Em outras palavras, as tecnologias sociais precisam 
estar fortemente direcionadas à capacidade de reconhecer os conhecimentos relevantes, adquiri-los, transformá-los e explorá-los (ZAHRA; GEORGE, 2002) nos processos produtivos.

Nesse sentido, Chaminade et al. (2009) defendem que uma capacidade central deve ser a possibilidade de experimentação por parte das firmas durante o processo de absorção de tecnologias. Isso significa dar menos peso às tradicionais políticas de incentivo à $P \& D$. Deve-se dar maior relevância às capacidades relacionadas a engenharia, design e capacidades gerenciais. Nessa direção, as políticas devem conferir atenção especial para o treinamento da mão de obra em capacidades básicas, engenharia avançada, design e capacitação gerencial e menos para subsídios a atividades de $\mathrm{P} \& \mathrm{D}$.

Um exemplo dessas diferenças foi observado por Mazzoleni e Nelson (2005) ao estudarem sistemas nacionais de países em emparelhamento. Os sistemas de treinamento acadêmico e de pesquisa pública (university-based) foram e continuam sendo uma estrutura institucional essencial no processo de emparelhamento. Porém, os autores notaram que as mudanças no ambiente econômico e na base científica e tecnológica das novas tecnologias favorecem a maior efetividade de programas de treinamento e pesquisa públicos do tipo application-oriented em engenharias e ciências, isto é, orientados para a resolução de problemas e o avanço de tecnologias de um bem definido grupo de usuários e comunidades.

Cimolli et al. (2007) observam que as políticas e as atividades provenientes de uma "engenharia institucional" afetam de forma conjunta três variáveis: i) capacidades tecnológicas e o ritmo em que organizações individuais e corporativas conseguem aprender; ii) sinais econômicos, como, por exemplo, de lucratividade; e iii) formas de interação entre as organizações e com as instituições não mercantis.

Os autores também destacam algumas sutis regularidades em alguns casos de sucesso de "engenharia institucional" e políticas em atingir maiores graus de desenvolvimento: i) centralidade das agências e políticas; ii) criação de uma estrutura de incentivos para agentes privados; iii) utilidade do mercado para retirar firmas com baixo desempenho, mas evitando eliminação de um grande conjunto de firmas; iv) medidas para limitação de comportamento rentista; e v) incorporação de novos e mais dinâmicos paradigmas tecnológicos.

A habilidade de criar instituições de forma planejada é muito mais limitada comparativamente à criação de tecnologias físicas. Portanto, tecnologias físicas são mais acessíveis por replicarem ou imitarem outras tecnologias físicas, diferentemente de tecnologias sociais. Estas últimas estão suscetíveis a caprichos e diferentes motivações políticas que raramente podem ser controlados.

\section{Considerações Finais}

As mudanças tecnológicas resultam em uma crise de ajustamento, demandando o surgimento de um novo design institucional e de novas tecnologias sociais. A capacidade dos países em responder às modificações tecnológicas passa a determinar o seu nível de inovação e, consequentemente, o seu grau de crescimento econômico. Como as instituições e as tecnologias sociais são influenciadas por fatores sociais e culturais, nem todos os países conseguem se adaptar a essas mudanças, pois os agentes podem considerar não desejável a adoção de determinados comportamentos. Apenas os países que conseguirem desenvolver um ambiente interno favorável ao desenvolvimento de novas instituições e capacitações sociais vão conseguir se adaptar à mudança tecnológica, auferindo crescimento econômico.

Como as instituições e as tecnologias sociais não se encontram no interior das firmas, sendo caracterizadas pela articulação entre diferentes agentes, podem ocorrer problemas de coordenação que impossibilitam a realização das mudanças institucionais, mesmo que essas sejam desejadas. A presença de problemas de coordenação implica que o mercado, deixado por si só, é incapaz de adaptar as modificações tecnológicas. Aqui surge espaço para a ação do Estado, através da elaboração de políticas públicas voltadas para a articulação entre os agentes e para a aquisição das novas instituições e tecnologias sociais demandadas, isto é, o Estado passa a ser demandado como agente responsável por eliminar os problemas de coordenação existentes. Dada a presença desses problemas, surge a necessidade de os países criarem sistemas nacionais de coordenação e inovação que estimulem e facilitem a absorção das novas tecnologias e a realização das modificações institucionais necessárias.

Assim, o crescimento econômico é explicado pela presença de características internas dos países que detêm elevada capacidade de adaptação e de elevada flexibilidade, passando pela presença de políticas públicas responsáveis por criar instituições e tecnologias sociais que elevam a capacidade de aprendizagem e adaptação das firmas, das instituições e das tecnologias sociais.

Portanto, a chave para o desenvolvimento passa a ser reformar instituições e tecnologias sociais para, assim, encorajar agentes a buscar novas tecnologias, sempre observando as particularidades de cada país ou sistema de inovação. As políticas públicas devem fazer com que as firmas, durante o processo de absorção de tecnologias, sejam induzidas à experimentação de novos processos e produtos. 
Porém, deve-se considerar o fato de que o Estado não é neutro ou descolado das demandas da sociedade. As políticas de coordenação para a inovação também passam por disputas políticas entre os grupos sociais, daí a necessidade de melhor compreensão do processo político e das instituições relacionadas, haja vista que as disputas políticas restringem e direcionam a ação do Estado, podendo desvirtuar esforços legítimos despendidos com o objetivo de promover a mudança tecnológica e o crescimento econômico.

A pergunta, portanto, não deve ser sobre quais são as instituições “certas”, mas quais instituições podem ser indutoras de trajetórias tecnológicas, dadas as idiossincrasias de cada sistema de inovação. Assim, fica claro que não é possível simplesmente transplantar instituições que dão certo em determinados lugares para outros. As tecnologias sociais estão "embedded" nas sociedades e nas firmas. Observar essa característica é um ponto de partida fundamental para determinar quais são as instituições que podem impelir os agentes ao processo inovativo.

\section{Referências}

AGHION, P.; HOWITT, P. Joseph Schumpeter lecture appropriate growth policy: A unifying framework. Journal of the European Economic Association, v. 4, n. 2/3, p. 269-314, 2006.

CHAMINADE, C.; VANG, J. Globalisation of knowledge production and regional innovation policy: Supporting specialized hubs in the Bangalore software industry. Research policy, v. 37, n. 10, p. 1684-1696, 2008.

CHAMINADE, C. et al. Designing innovation policies for development: Towards a systemic experimentationbased approach. Handbook of Innovation Systems and Developing Countries: Building domestic capabilities in a global setting, p. 360-379, 2009.

CIMOLI, M. et al. Instituições e políticas moldando o desenvolvimento industrial: uma nota introdutória. Revista brasileira de inovação, v. 6, n. 1, p. 55-85, 2007.

COHEN, W. M. et al. Routines and other recurring action patterns of organizations: Contemporary research issues. Industrial and Corporate Change, Oxford, v. 5, n. 3, p. 653-698, 1996.

CONCEIÇÃO, O. A. C. Instituições e crescimento econômico: da "tecnologia social” de Nelson à "causalidade vebleniana” de Hodgson. In: ENCONTRO NACIONAL DE ECONOMIA, 37., 2009, Foz do Iguaçu, PR. Anais... Foz do Iguaçu: ANPEC, 2009. 1 CD-ROM.

CONCEIÇÃO, O. A. C. Há compatibilidade entre a “tecnologia social” de Nelson e a "causalidade vebleniana” de Hodgson? Brazilian Journal of Political Economy, v. 32, n. 1, p. 109-127, 2012.

CORDEN, W. M. Relationships between macroeconomic and industrial policies. The World Economy, v.3, n. 2, p. $167-184,1980$.

DOSI, G. Sources, procedures, and microeconomic effects of innovation. Journal of economic literature, p. 1120-1171, 1988.

DOSI, G.; NELSON, R. R. An introduction to evolutionary theories in economics. Journal of evolutionary economics, v. 4, n. 3, p. 153-172, 1994.

DOSI, G.; NELSON, R.; WINTER, S. Introduction: The nature and dynamics of organizational capabilities. Oxford: Oxford University, 2000.

FELDMAN, M. Organizational routines as a source of continuous change. Organization Science, Maryland, v. 11, n. 6, p. 611-629, Nov./Dec. 2000.

FOSS, N. Bounded rationality and tacit knowledge in the organizational capabilities approach: An assessment and a re-evaluation. Industrial and Corporate Change, n. 12, p. 185-201, 2003.

FREEMAN, C.; PEREZ, C. Structural crises of adjustment: business cycles. Technical change and economic theory. London: Pinter, 1988. 
JOHNSON, C. Introduction: The idea of industrial policy. In: Francisco, CA: ICS Press, 1984.

(Ed.). The Industrial Policy Debate. San

KESTING, P. The relation between routine and decision: An action-based approach. Unpublished draft.

LANGLOIS, R.; ROBERTSON, P. Firms, markets and economic change: A dynamic theory of business institutions. London/New York: Routledge, 1995.

MAZZOLENI, R.; NELSON, R. R. The roles of research at universities and public labs in economic catch-up. LEM Working Paper Series, 2005.

NELSON, R. R. Recent evolutionary theorizing about economic change. Journal of economic literature, v. 33, n. 1, p. 48-90, 1995.

NELSON, Richard R. Bringing institutions into evolutionary growth theory. In: Social Institutions and Economic Development. Springer, Dordrecht, 2002. p. 9-12.

NELSON, R. R. What makes an economy productive and progressive? What are the needed institutions? LEM Working Paper Series, 2006.

NELSON, R. R. What enables rapid economic progress: What are the needed institutions? Research policy, v. 37, n. 1, p. 1-11, 2008.

NELSON, R. R.; DOSI, G. An introduction to evolutionary theories in economics. Journal of evolutionary economics, v. 4, n. 3, p. 153-172, 1994.

NELSON, R. R.; SAMPAT, B. N. Making sense of institutions as a factor shaping economic performance. Revista de Economía Institucional, v. 3, n. 5, p. 17-51, 2001.

NELSON, R. R.; WINTER, S. G. Evolutionary theorizing in economics. Journal of Economic Perspectives, v. 16, n. 2, p. 23-46, 2002.

NELSON, Richard R.; WINTER, Sidney G. Uma teoria evolucionária da mudança econômica. Editora Unicamp, 2005.

PENTLAND, B.; RUETER, H. Organizational routines as grammars of action. Administrative Science Quarterly, New York, v. 39, p. 484-510, 1994.

PEREZ, C. Technological revolutions, paradigm shifts and socio-institutional change. Globalization, economic development and inequality: An alternative perspective, p. 217-242, 2004.

SCHUMPETER, J. A. Change and the Entrepreneur. Essays of JA Schumpeter, 1934.

TEECE, D.; PISANO, G.; SHUEN, A. Dynamic capabilities and strategic management. Strategic Management Journal, New Jersey, v. 18, n. 7, p. 509-533, Aug. 1997.

TIGRE, P. B. Inovação e teorias da firma em três paradigmas. Revista de Economia Contemporânea, v. 3, p. 67111, 1998.

TOREZANI, T. A. Desenvolvimento econômico, instituições e inovação: a interação entre as abordagens institucionalista e evolucionária. Perspectiva Econômica, v. 10, n. 2, p. 67-79, 2014.

ZAHRA, S.; GEORGE, G. Absorty Capacity: A review reconceptualization and extension. Academic Management Review, p. 185-203, 2002.

ZOLLO, M.; WINTER, S. Deliberate learning and the evolution of dynamics capabilities. Organization Science, Maryland, v. 13, n. 3, p. 339-351, 2002. 